\title{
Zoster prophylaxis after allogeneic hematopoietic cell transplantation using acyclovir/valacyclovir followed by vaccination
}

\author{
Kareem Jamani, ${ }^{1}$ Judy MacDonald, ${ }^{2,3}$ Martin Lavoie, ${ }^{4}$ Tyler S. Williamson, ${ }^{2}$ Christopher B. Brown, ${ }^{1}$ Ahsan Chaudhry, ${ }^{1}$ \\ Victor H. Jimenez-Zepeda, ${ }^{1}$ Peter Duggan, ${ }^{1}$ Jason Tay, ${ }^{1}$ Douglas Stewart, ${ }^{1}$ Andrew Daly, ${ }^{1}$ and Jan Storek ${ }^{1}$ \\ ${ }^{1}$ Alberta Blood and Marrow Transplant Program, University of Calgary and Alberta Health Services, Calgary, AB, Canada; ${ }^{2}$ Department of Community Health Sciences, \\ Cumming School of Medicine, University of Calgary, Calgary, AB, Canada; ${ }^{3}$ Population, Public and Aboriginal Health, Alberta Health Services, Calgary, AB, Canada; and \\ ${ }^{4}$ Alberta Health, Government of Alberta, Edmonton, AB, Canada
}

\section{Key Points}

- Two years of antiviral prophylaxis and VZV vaccination reduce the incidence of VZV disease and nearly eliminate post-herpetic neuralgia.
Varicella zoster virus (VZV) disease (usually cutaneous zoster) occurs frequently after hematopoietic cell transplantation (HCT), and postherpetic neuralgia (PHN) results in poor quality of life. The optimal prophylaxis of VZV disease/PHN has not been established. At our center, before 2008 , VZV prophylaxis consisted of $\sim 1$ year of post-HCT acyclovir/valacyclovir (“old strategy"), whereas post-2008 prophylaxis consisted of 2 years of acyclovir/ valacyclovir followed by immunization using varicella vaccine ("new strategy"). We performed a retrospective study comparing the cumulative incidence of VZV disease and PHN among patients who completed the old strategy $(n=153)$ vs the new strategy $(n=125)$. Patients who completed the old strategy had a significantly higher cumulative incidence of VZV disease ( $33 \%$ vs $17 \%$ at 5 years, $P \leq .01$ ) and PHN ( $8 \%$ vs $0 \%$ at 5 years, $P=.02$ ). In conclusion, VZV prophylaxis with 2 years of acyclovir/valacyclovir followed by vaccination appears to result in a low incidence of VZV disease and may eliminate PHN.

\section{Introduction}

Varicella zoster virus (VZV) disease occurs in 17\% to 80\% (median 33\% based on 13 studies reviewed by $\mathrm{Ho}$ and Arvin $^{1}$ ) of allogeneic hematopoietic cell transplant (allo-HCT) recipients. In the vast majority of adults, VZV disease post allo-HCT occurs as a result of reactivation of latent infection after childhood primary infection (chicken pox). ${ }^{2}$ Post allo-HCT, VZV disease typically presents as a dermatomal rash (shingles), but disseminates on the skin in $15 \%$ to $30 \%$ of cases. ${ }^{3,4}$ In rare cases, VZV infection may involve the viscera or the central nervous system, a situation that is difficult to recognize and often fatal. ${ }^{5}$ Despite treatment, VZV disease leads to postherpetic neuralgia (PHN) in $25 \%$ to $68 \%$ (median $32 \%{ }^{6-8}$ ) of HCT recipients who develop dermatomal zoster. ${ }^{1}$

Four randomized ${ }^{9-12}$ and 1 retrospective study ${ }^{13}$ have demonstrated that the frequency of VZV disease is reduced during prophylaxis with acyclovir post allo-HCT; however, a rebound effect is observed in prophylaxed patients, who frequently develop VZV disease after discontinuation of acyclovir. As a result, the cumulative incidence of VZV disease is similar to the nonprophylaxed groups. In contrast, 1 large nonrandomized study showed a significant reduction in VZV disease with 1 year of acyclovir prophylaxis without a rebound effect ${ }^{14}$; however, the result of this study may have been affected by insufficient follow-up. As a result, there is no consensus on VZV prophylaxis strategy, with significant variation in practice across transplant centers. ${ }^{15}$

VZV-specific T-cell immunity, even in the absence of VZV prophylaxis, does not reconstitute post-allo$\mathrm{HCT}$ in the absence of a clinical VZV reactivation event. ${ }^{16}$ This finding is in keeping with the rebound
Submitted 24 August 2016; accepted 16 October 2016. DOI 10.1182/ bloodadvances. 2016000836

Presented in abstract form at the 57th annual meeting of the American Society of Hematology, Orlando, FL, 5 December 2015.
The full-text version of this article contains a data supplement. (c) 2016 by The American Society of Hematology 
effect described above and suggests that a prophylaxis strategy that includes vaccination is required. There are data supporting the safety and efficacy of the varicella vaccines in VZV-seronegative pediatric allo-HCT recipients, with the most common toxicity being a self-limited disseminated vesicular rash that occurs in $<10 \%$ of patients 2 to 6 weeks postvaccine. ${ }^{17-19}$ There are no published reports on the use of the live attenuated varicella vaccine in adults and no comparisons of the efficacy of a vaccine vs nonvaccine prophylaxis strategy. International guidelines list the live attenuated varicella vaccine as optional in patients $>2$ years after transplant who do not have active graft-versus-host disease (GVHD) and are not on immunosuppression. ${ }^{20}$

At our center, patients received $\sim 12$ months of acyclovir (400 mg twice a day) or valacyclovir (500 mg daily) as VZV prophylaxis postHCT. Longer courses were used in the setting of immunosuppression for GVHD. In 2008, in an effort to reduce the incidence of VZV disease and $\mathrm{PHN}$, institutional guidelines were developed that recommend 24 months of acyclovir (400 $\mathrm{mg}$ twice a day) or valacyclovir (500 $\mathrm{mg}$ daily), followed by 2 doses of varicella vaccine 3 months apart (Varivax; Merck, Kenilworth, NJ, or Varilrix; GlaxoSmithKline, Brentford, United Kingdom). For patients who require immunosuppression beyond 24 months, acyclovir/valacyclovir is continued until at least 3 months after discontinuation of immunosuppressive therapy and is followed by 2 doses of the varicella vaccine. Acyclovir/valacyclovir is discontinued 1 day prior to the first dose of the vaccine and not restarted thereafter. Here, we retrospectively compared the patients who completed the strategy of $\sim 1$ year of acyclovir/valacyclovir and no vaccination ("old strategy") with those who completed the strategy of $\geq 2$ years of acyclovir/valacyclovir followed by vaccination ("new strategy").

\section{Methods}

\section{Patients}

The study protocol was approved by the Health Research Ethics Board of Alberta. Patients were identified from a dedicated comprehensive database of all patients receiving allo-HCT in Alberta. Consecutive patients receiving a first allo-HCT between January 1, 2003 and December 31, 2011 were reviewed. January 1,2003 was chosen given that electronic medical records became reliably available in Alberta at that time, while the December 31, 2011 transplant date was chosen to allow for adequate follow-up as of April 30, 2016. Patient medical records, including medication records and vaccination records, were reviewed and supplemented by provincial (Albertan) vaccine-related adverse event database. Transplant physicians cleared patients for varicella vaccination using all of the following eligibility criteria: $\geq 24$ months post-HCT, off systemic immunosuppression for $\geq 3$ months, no active GVHD, and, if applicable, off intravenous immune globulin (IVIG) for $\geq 8$ months (or $\geq 10$ months if $\geq 1 \mathrm{mg} / \mathrm{kg}$ dosing of IVIG). ${ }^{21}$

Patients were included in the "new strategy" group if they received acyclovir, valacyclovir, or another VZV active antiviral drug until at least 22 months post-HCT or, in the case of patients treated with immunosuppressive therapy beyond 22 months post-HCT, until at least 3 months postdiscontinuation of the last immunosuppressive drug, followed by at least 1 dose of varicella vaccine. Patients were included in the "old strategy" group if they completed any duration of acyclovir, valacyclovir, or another VZV active antiviral drug without subsequent vaccination. During the adoption period of the new guidelines, some patients received $<22$ months of prophylaxis but were subsequently vaccinated at $\geq 24$ months post-HCT. These patients were included in the old strategy group but were censored at the time of vaccination. Patients who reached end of follow-up without discontinuing acyclovir/valacyclovir were included in a separate group ("never off antivirals" group).

\section{Transplantation}

The usual conditioning regimen during the study period was fludarabine ( $\left.250 \mathrm{mg} / \mathrm{m}^{2}\right)$, busulfan $(\sim 12.8 \mathrm{mg} / \mathrm{kg} \mathrm{IV}$, pharmacokinetically adjusted), and antithymocyte globulin (Thymoglobulin; Sanofi, Paris, France; $4.5 \mathrm{mg} / \mathrm{kg}$ ), ${ }^{22}$ with or without total body irradiation (4 Gy). ${ }^{23}$ Additional GVHD prophylaxis was provided as methotrexate (days 1, 3, 6, 11) and cyclosporine (until 3-6 months post-HCT). Prophylaxis for HSV and VZV with acyclovir $400 \mathrm{mg}$ twice a day or valacyclovir $500 \mathrm{mg}$ daily was started by day +1 . Grade 2-4 acute GVHD (Consensus criteria) ${ }^{24}$ and extensive (Seattle criteria) ${ }^{25}$ or moderate-severe (National Institutes of Health criteria) ${ }^{26}$ chronic GVHD were treated with corticosteroids with or without additional immunosuppressive drugs. IVIG was not routinely used post-HCT; rather, it was reserved for a minority of patients who developed recurrent or severe infections in the setting of low immunoglobulin G level.

\section{Endpoints}

Endpoints were VZV disease and $\mathrm{PHN}$ as documented in physician progress notes. VZV disease was categorized as dermatomal (involvement of 1-2 dermatomes) or disseminated (involvement of $>2$ dermatomes or extracutaneous involvement). PHN was defined as pain persisting in the affected dermatome(s) for $>3$ months after the onset of rash. ${ }^{27}$ Patients were followed until the first episode of VZV disease, competing risk event (see Statistics), or last clinic visit, whichever occurred first. Other outcomes of interest were adverse events due to VZV prophylaxis, cases of resistant VZV and resistant herpes simplex virus (HSV), and cases requiring inpatient treatment of VZV disease.

\section{Statistics}

The cumulative incidences of VZV disease and PHN were compared between the 2 groups using the Fine-Gray test. Covariates included donor type (HLA matched sibling vs other), graft type (filgrastim mobilized blood stem cells vs other), and significant GVHD (grade 2-4 acute GVHD or chronic GVHD needing systemic immunosuppression occurring at any time before the end of follow-up vs no significant GVHD). Type of antiviral drug used (acyclovir vs valacyclovir) was not included as a covariate as these drugs appear to be equally effective during prophylaxis ${ }^{28}$ and are likely irrelevant after prophylaxis is discontinued. Competing risk events were death and events that increase risk of infection, including relapse, graft failure, and second malignancy (excluding posttransplant lymphoproliferative disorder, carcinoma in situ, and nonmelanoma skin cancer). Statistical analyses were performed on STATA 14.1 (StataCorp, College Station, TX).

\section{Results}

\section{Patient characteristics}

During the study period, 588 patients underwent first allo-HCT (Figure 1). Thirty patients were excluded from analysis: 17 patients were lost to follow-up before 6 months posttransplant and 13 patients had inadequate chart data (unable to determine VZV disease prophylaxis strategy). Of the remaining 558 patients, 280 could not be followed for VZV disease/PHN after acyclovir/valacyclovir discontinuation as they reached end of follow-up before discontinuation of acyclovir/valacyclovir (never off antivirals group). Of the remaining 278 patients, 153 were treated with the old strategy and 125 were treated with the new strategy. Three patients were inappropriately cleared for vaccination: 1 was vaccinated while on $30 \mathrm{mg}$ prednisone and 2 were vaccinated too close to the last IVIG dose ( 8 days and 7 months since last dose). Nineteen of the new strategy patients received only 1 of the 2 recommended doses of the varicella vaccine and 3 took acyclovir/ valacyclovir beyond the first day of vaccination. Patient characteristics for the old strategy and the new strategy groups are shown in Table 1. For the never off antivirals group, patient characteristics and reasons for reaching end of follow-up are presented in supplemental Tables 1 


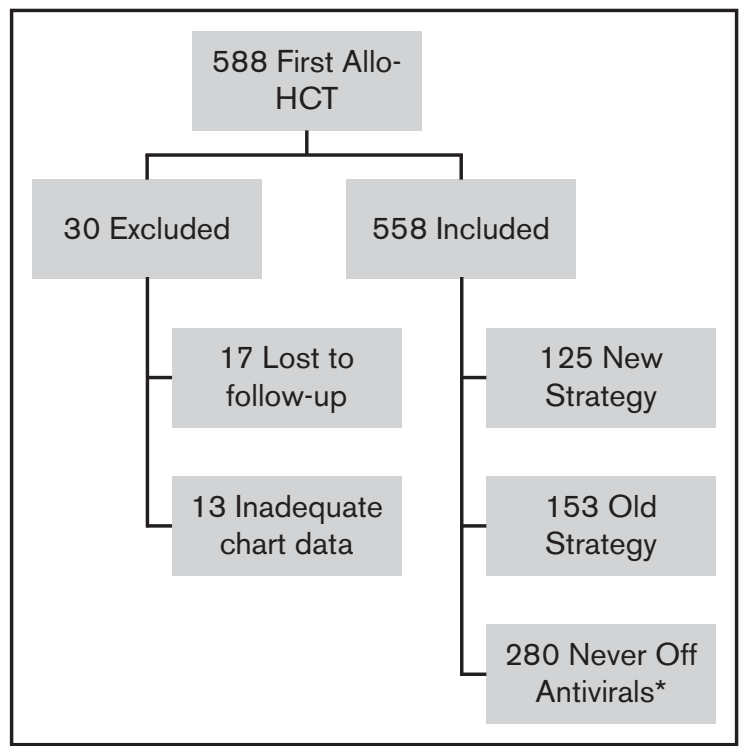

Figure 1. Flow diagram of patients receiving first allo-HCT in Calgary 20032011 reviewed for the study. *Did not complete acyclovir/valacyclovir before end of follow-up.

and 2. The number of patients in each group by transplant year is shown in supplemental Table 3 and reveals that uptake of the new strategy was a gradual process between transplant year 2006 and 2011.

\section{Duration of acyclovir/valacyclovir administration and follow-up}

The median duration of acyclovir/valacyclovir administration after transplant was 384 days (range 48-2350) in the old strategy group. Patients in the new strategy group were cleared by their transplant physician for varicella vaccination at a median of 750 (range 6623626) days post-HCT (this was the last documented day of acyclovir/ valacyclovir use). Patients then had to self-present to a public health clinic for vaccination and were asked to discontinue acyclovir/ valacyclovir the day before vaccination. Median time to first varicella vaccination was 783 days (691-3633) post-HCT.

Median follow-up post-HCT was 1811 days (range 224-4523) and 2119 days (range 763-4339) in the old and new strategy groups, respectively. Median follow-up post-HCT in the never off antivirals group was 168 days (range 8-4607).

Median follow-up from discontinuation of acyclovir/valacyclovir was 1256 days (range 34-4060) in the old strategy group. In the new strategy group, median follow-up from first vaccination was 1072 days (range 6-3152).

\section{VZV disease}

Patients in the old strategy group had a significantly higher cumulative incidence of VZV disease compared with those in the new strategy group (sub-hazard ratio $[\mathrm{SHR}]=2.5 ; P=.0002$ ) (Figure 2). Donor type, graft type, and significant GVHD were not associated with VZV disease (Table 2). The cumulative incidence of VZV disease at 5 years post-HCT was 33\% (51/153) in the old and $17 \%(21 / 131)$ in the new strategy group. For comparison, 4 of 280 (1\%) patients from the never off antivirals group developed VZV disease by 5 years. There was evidence of a rebound effect in the old strategy group: among patients who received 11-13 months of acyclovir/valacyclovir without subsequent vaccination $(n=39)$, the cumulative incidence of VZV disease was $3 \%$ at 1 year, $38 \%$ at 2 years, and $46 \%$ at 5 years post-HCT. In contrast, among the new strategy patients who received vaccination between 22 and 26 months post-HCT ( $n=67$ ), the cumulative incidence of VZV disease was $6 \%$ at 2 years, $13 \%$ at 3 years, and $18 \%$ at 6 years post-HCT.

In the old strategy group, by the end of follow-up, 7 VZV disease events occurred while on acyclovir/valacyclovir, and the remaining 49 events occurred after acyclovir/valacyclovir discontinuation. In the new strategy group, by the end of follow-up, $7 \mathrm{VZV}$ disease events occurred while on acyclovir/valacyclovir and the remaining 16 events occurred after the first vaccine dose. Of the 16 postvaccine events, 4 occurred between 2 and 6 weeks and 12 occurred beyond 6 weeks postvaccination. Of the 4 events between 2 and 6 weeks, 2 presented as disseminated skin disease ( 1 with corneal involvement that resolved promptly with topical therapy) and 2 presented in a dermatomal distribution. Of the 12 events beyond 6 weeks, 5 patients deviated from protocol: 2 patients took acyclovir/valacyclovir through vaccination; 2 received only 1 dose of the vaccine; and 1 was vaccinated while on $30 \mathrm{mg}$ prednisone. In 1 patient, VZV genotyping was available; this patient developed disseminated skin disease 18 days after first vaccine, and the genotyping revealed vaccine (Oka) strain VZV.

Disseminated VZV disease occurred in 8 patients in each group (Table 3). By the end of follow-up, 11 patients in the old strategy group (7.2\%) and 2 patients in the new strategy group (1.6\%) required inpatient treatment of VZV disease. The most common reasons for inpatient treatment were severe skin pain or visceral disease. Treatment of patients who developed VZV disease was with high-dose acyclovir, valacyclovir, or famciclovir, except for 2 patients in the old strategy group (in whom treatment was not specified) and 2 patients in the new strategy group (1 who presented after all lesions were crusted and 1 for whom treatment was not specified).

\section{Post-herpetic neuralgia}

The old strategy patients had a significantly higher cumulative incidence of PHN compared with the new strategy patients (SHR 13.4, $P=.02$ ) (Figure 3). Donor type, graft type, and significant GVHD were not associated with PHN (Table 2). A total of 13 patients (8.5\%) in the old strategy group and no patients in the new strategy group developed PHN by 5 years post-HCT. One patient $(0.4 \%)$ in the never off antivirals group developed PHN by 5 years.. In the old strategy group, by the end of follow-up, all 14 PHN events occurred after discontinuation of acyclovir/valacyclovir. In the new strategy group, 1 patient developed $\mathrm{PHN}$ at 9 years post-HCT.

\section{Resistant VZV and HSV}

One patient in the old strategy group had suspected acyclovirresistant VZV, with persistent neurological symptoms and cerebrospinal fluid VZV polymerase chain reaction positivity, and responded to foscarnet. No patients in the new strategy group had a suspected or documented resistant VZV. In the old strategy group, 3 patients (2\%) developed HSV that was clinically suspected to be resistant to acyclovir-2 were treated with foscarnet and 1 with famciclovir. In the new strategy group, 2 patients (1.6\%) developed resistant HSV, 


\begin{tabular}{|c|c|c|c|}
\hline & Old strategy $(N=153)$ & New strategy $(\mathrm{N}=125)$ & $\boldsymbol{P}^{*}$ \\
\hline Median age (range) & $46(17-65)$ & $50(18-66)$ & .42 \\
\hline Recipient VZV seropositive (\%) & $66(99)+$ & $101(98) \neq$ & .71 \\
\hline Donor VZV seropositive (\%) & $65(100) \dagger$ & $101(98) \neq$ & .69 \\
\hline \multicolumn{4}{|l|}{ VZV prophylaxis drug (\%) } \\
\hline Acyclovir & $137(90)$ & $65(52)$ & $<.01$ \\
\hline Valacyclovir & $16(10)$ & $60(48)$ & \\
\hline \multicolumn{4}{|l|}{ Underlying disease (\%) } \\
\hline Acute leukemia & $84(55)$ & $75(60)$ & .85 \\
\hline MDS & $19(12)$ & $11(9)$ & \\
\hline CLL/lymphoma & $26(17)$ & $22(18)$ & \\
\hline CML & $10(7)$ & 7 (5) & \\
\hline Other & $14(9)$ & $10(8)$ & \\
\hline \multicolumn{4}{|l|}{ Donor§ (\%) } \\
\hline Sibling & $87(57)$ & $51(41)$ & .02 \\
\hline MUD & $48(31)$ & $58(46)$ & \\
\hline MMUD & $16(11)$ & $16(13)$ & \\
\hline Haploidentical & $2(1)$ & $0(0)$ & \\
\hline \multicolumn{4}{|l|}{ Stem cell source (\%) } \\
\hline Peripheral blood & $127(83)$ & $120(96)$ & $<.01$ \\
\hline Bone marrow & $23(15)$ & $4(3)$ & \\
\hline Umbilical cord & $3(2)$ & $1(1)$ & \\
\hline \multicolumn{4}{|l|}{ Conditioning (\%) } \\
\hline FluBuATG & $53(35)$ & $36(29)$ & .24 \\
\hline FluBuATG + TBI & $89(58)$ & $84(67)$ & \\
\hline Other\| & $11(7)$ & $5(4)$ & \\
\hline \multicolumn{4}{|l|}{ GVHD prophylaxis (\%) } \\
\hline $\mathrm{CSA}+\mathrm{MTX}$ & $147(96)$ & $122(98)$ & .71 \\
\hline Other 9 & $6(4)$ & $3(2)$ & \\
\hline \multicolumn{4}{|l|}{ Acute GVHD (\%) } \\
\hline None & $83(54)$ & $59(47)$ & .36 \\
\hline 1 & $35(23)$ & $37(30)$ & \\
\hline ॥ & $25(16)$ & $25(20)$ & \\
\hline III & $9(6)$ & $3(2)$ & \\
\hline IV & $1(1)$ & $1(1)$ & \\
\hline \multicolumn{4}{|l|}{ Chronic GVHD (\%) } \\
\hline None & $109(71)$ & $67(54)$ & $<.01$ \\
\hline No systemic Rx & $19(12)$ & $19(15)$ & \\
\hline Systemic Rx & $25(16)$ & 39 (31) & \\
\hline \multicolumn{4}{|l|}{ Significant GVHD\# (\%) } \\
\hline No & $93(61)$ & $57(46)$ & .02 \\
\hline Yes & $60(39)$ & $68(54)$ & \\
\hline
\end{tabular}

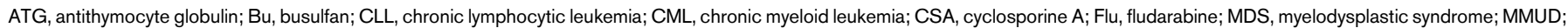
mismatched unrelated donor; MTX, methotrexate; MUD, matched unrelated donor; Rx, treatment; TBI, total body irradiation.

${ }^{*}$ For ordinal variables, Mann-Whitney $U$ test was used, and for nominal variables, $\chi^{2}$ or Fisher's exact test was used as appropriate, using SigmaPlot 13 (Systat, San Jose, CA).

tOf 67 recipients or 65 donors for whom VZV serostatus was available.

¥Of 103 recipients and donors for whom VZV serostatus was available.

$\S$ Sibling = HLA-matched sibling; MUD = 8/8 allele-matched unrelated donor; MMUD (mismatched unrelated donor) included 6-7/8 allele-matched unrelated donors.

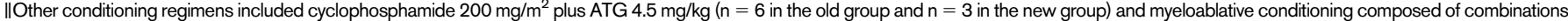

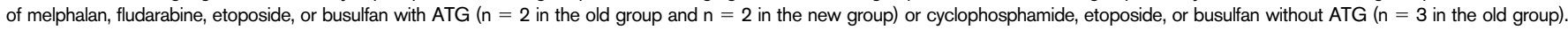

TOther included cyclosporine (CSA) alone ( $n=4$ in the old group), prednisone ( $n=1$ in the old group and $n=2$ in the new group), and mycophenolate ( 1 in each group).

\#Grades 2-4 acute GVHD and chronic GVHD requiring immunosuppression. 


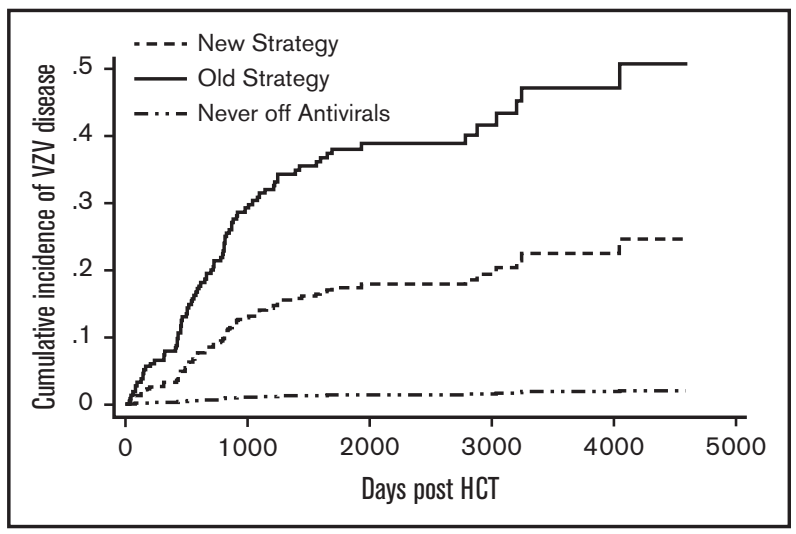

Figure 2. Cumulative incidence of VZV disease. The difference between the new strategy and the old strategy groups was significant $(P \leq .01)$. The never off antivirals group consisted of patients who were on acyclovir/valacyclovir until the end of follow-up.

both documented by HSV genotyping. One was treated successfully with foscarnet and 1 case resolved spontaneously.

\section{Safety}

Four patients (2.6\%) in the old strategy group had a documented adverse event whereby antiviral therapy was considered as a potential cause: 1 patient developed itching; 1 patient developed nausea; 1 patient developed irritability; and 1 patient developed hepatitis on valacyclovir, which remained undiagnosed but had a differential diagnosis of drug-induced hepatitis (dapsone vs valacyclovir) vs a non-ABC viral hepatitis. In the new strategy group, 3 patients $(2.4 \%)$ had adverse events. All were considered related to vaccination: 1 unspecified allergic reaction to the first varicella vaccine dose (however, the patient subsequently received a second vaccine dose without reported adverse event); 1 localized rash; and 1 pain/swelling at injection site.

\section{Discussion}

To our knowledge, this is the first report of the use of the live attenuated varicella vaccine in adult HCT recipients. One previous study reported on the use of the shingles vaccine (which contains more virus particles than the varicella vaccine) in 58 adult allo-HCT recipients; however, the antiviral prophylaxis preceding the vaccination was not given, follow-up was relatively short (9.5 months postvaccination), and there was no comparator group. Our retrospective, single-center analysis provides a cohort of uniformly treated patients except for the institutional VZV prophylaxis guideline change that has allowed for the comparison of outcomes between the 2 strategies. The median duration of prophylaxis in the old strategy group was $\sim 1$ year, a relatively common duration of VZV prophylaxis among North American HCT centers. ${ }^{14,15}$ The old and new strategy groups were balanced with respect to age, underlying disease, VZV serostatus, and conditioning regimen. In addition, the length of follow-up after discontinuation of antivirals was similar in both groups. However, patients in the new strategy group were less likely to have a matched sibling donor and more likely to receive a peripheral blood stem cell graft and to develop chronic GVHD requiring systemic immunosuppression (Table 1). The higher incidence of chronic GVHD is possibly a result of the increased numbers of unrelated donors and peripheral blood stem cell grafts in the new strategy group. ${ }^{29,30}$ The imbalance between the 2 groups in donor type, graft type, and significant GVHD incidence was dealt with by including the donor type, the graft type, and the significant GVHD as covariates in our multivariate analyses. Multivariate analysis was particularly important for GVHD, the only well-documented risk factor for VZV disease after HCT. ${ }^{6,31}$ Nevertheless, contrary to the published reports, we did not find significant GVHD to be associated with VZV disease or PHN, and this is likely because our patients in both groups remained on acyclovir/valacyclovir while on immunosuppression.

The most important finding of our study is that PHN incidence is significantly reduced with the new strategy. The reduced incidence of PHN is highly encouraging as PHN is a difficult-to-treat, chronic condition that significantly impacts quality of life. ${ }^{32,33}$ There are no studies of the effect of varicella vaccination on the incidence of PHN in any population. However, the shingles vaccine is known to reduce $\mathrm{PHN}$ incidence in older, nontransplant populations. ${ }^{34}$

The new strategy also resulted in a lower cumulative incidence of VZV disease. Similar to published reports, we noted a rebound effect in those receiving 1 year of acyclovir/valacyclovir in the old strategy group: a significant number of patients developed VZV disease after acyclovir/valacyclovir discontinuation. ${ }^{9-13}$ Interestingly, a significant rebound was not noted in the new strategy group; however, post-acyclovir/valacyclovir discontinuation VZV disease events did occur. We suggest that these likely included events of vaccine-strain VZV disease, although this was only evaluated and confirmed by genotype testing in 1 patient. It is known that VZV disease 2 to 6 weeks postvaccination is most often due to vaccine strain VZV, ${ }^{35}$ and 4 of 16 postvaccine events in the new strategy group occurred in this timeframe. These events tend to present as disseminated skin disease or injection site vesicles and typically follow a mild clinical course, both in healthy children and in children after allo-HCT. ${ }^{17,35}$ Consistent with this, none of our 4 adult patients developed PHN or required hospitalization.

The reduction in incidence of VZV disease with the new strategy was also despite inclusion of events associated with deviation from the protocol (discontinuing acyclovir/valacyclovir after the first vaccine dose, receiving only 1 dose of the vaccine and vaccination while on immunosuppression). Such deviations from protocol

Table 2. Results of multivariate analysis for the cumulative incidence of VZV disease and PHN

\begin{tabular}{lcr}
\hline & SHR (95\% Cl) & $\boldsymbol{P}$ \\
\hline Cumulative incidence of VZV & & \\
\hline Old strategy (vs New strategy) & $2.5(1.5-4.1)$ & $<.01$ \\
\hline Significant GVHD* (yes/no) & $0.8(0.5-1.3)$ & .37 \\
\hline PBSC (vs other graft type) & $0.9(0.7-1.3)$ & .64 \\
\hline Matched sibling (vs other donor type) & $0.9(0.6-1.4)$ & .60 \\
\hline Cumulative incidence of PHN & & .02 \\
\hline Old strategy (vs New strategy) & $13.4(1.5-120.8)$ & .56 \\
\hline Significant GVHD* (yes/no) & $1.4(0.5-3.8)$ & .70 \\
\hline PBSC (vs other graft type) & $0.8(0.2-2.7)$ & .90 \\
\hline Matched sibling (vs other donor type) & $0.9(0.3-2.7)$ & \\
\hline Cl, confidence interval. & & \\
*Significant GVHD onset preceded VZV disease onset in all patients in the new and old \\
strategy groups.
\end{tabular}


Table 3. Type of VZV disease in each group at the end of follow-up

\begin{tabular}{lcc}
\hline Type of VZV disease & Old strategy & New strategy \\
\hline Dermatomal & 48 & 15 \\
\hline Disseminated skin & 3 & 7 \\
Opthalmic $^{*}$ & 3 & 1 \\
Visceral $^{*}$ & 1 & 0 \\
CNS $^{*}$ & 1 & 0 \\
\hline Total VZV disease cases & 56 & 23 \\
\hline
\end{tabular}

CNS, central nervous system.

*These patients also had skin disease.

illustrate the challenges of instituting a new prophylaxis strategy and may have influenced the results of our study. For example, it is important to educate HCT/vaccination clinic staff and patients that acyclovir/valacyclovir must be discontinued 1 day before vaccination so as to not kill the vaccine strain VZV, rendering the vaccine ineffective. In addition, in Alberta, vaccines are administered at public health clinics, and thus, once cleared for vaccination in the transplant clinic, patients must self-present to the public health clinics. Perhaps due to this, 19 (15\%) patients presented for only 1 dose of the 2-dose vaccine series, and this is known to be associated with primary vaccine failure. ${ }^{36}$ Twelve $(9.6 \%)$ patients in the new strategy group experienced vaccine failure, developing VZV disease beyond 6 weeks postvaccination. Five of these were known to have deviated from the protocol. Of the 12 patients, none had dissemination outside of the skin; only 1 required hospitalization, and only 1 developed $\mathrm{PHN}$. This low rate of complications is consistent with reports of $\sim 95 \%$ varicella vaccine efficacy in healthy children who receive 2 doses of the vaccine and almost complete elimination of varicella-related complications in vaccinated children. ${ }^{37}$ Adults are known to have less effective cell-mediated immune responses to varicella vaccination than children, and this might additionally explain the vaccine failure we observed. ${ }^{38}$

That we could not perform detailed immunologic studies before and after vaccination given the retrospective nature of this study is a limitation. There are no prospective controlled studies to guide the timing of live vaccination post-HCT and, thus, recommendations are based on expert opinion. Our clinical clearance criteria are in line with the 2009 international post-HCT vaccination guidelines ${ }^{39}$ and have the advantage of being simple. Other experts have suggested specific immunologic parameters prevaccination, most commonly a CD4 count $>200$ cells/ $\mu L^{15,17}$ It is likely that the vast majority of patients in our new strategy group had a CD4 count $>200$ cells $/ \mu \mathrm{L}$ before vaccination as we have previously shown that the median CD4 count at 2 years post-HCT at our center is 345 cells $/ \mu \mathrm{L}$ (25th75th percentile, 267-443). ${ }^{40}$ From a postvaccination perspective, we do not routinely assess anti-VZV antibody titers at our center because they are known to correlate poorly with risk of VZV reactivation. ${ }^{41-43}$ In contrast, it would certainly be informative to prospectively assess cell-mediated immune response to VZV serially postvaccination to investigate what factors might be associated with poor or waning immune response and occurrence of VZV disease after vaccination. However, these immunologic data would arguably not change our current practice given that we are left with an extremely low rate of PHN (the most important endpoint from a clinical perspective).
Additional limitations of our study include that a retrospective study cannot account for secular trends that may have occurred over the time period under review inherent in such a study design. Nevertheless, an institutional guideline change is responsible for the creation of 2 groups, and this reduces the chance that there was selection bias at play. Moreover, the 2 cohorts were managed similarly, and our multivariate analysis took into account imbalances in factors that could influence the risk of VZV disease or PHN, further reducing the risk of bias. Nevertheless, the large number of patients included in the never off antivirals group could have led to selection bias as it is not clear how many of these patients would have been placed in the old or new strategy groups in an intentionto-treat-type analysis. The lack of an intention-to-treat analysis reinforces that our findings should be confirmed in a prospective study. Second, the retrospective nature of our study does raise the possibility of incomplete information and misdiagnosis. We attempted to minimize selection bias by including all consecutive patients assessed at our institution. Indeed, our institution has implemented organizational strategies to capture detailed and complete information in more recent years, further reducing the risk of missing VZV event documentation in the new strategy group. Third, we were not able to determine whether the lower incidence of VZV disease and PHN was due to the longer duration of acyclovir/valacyclovir, vaccination, or both. However, given that VZV-specific immunity does not recover spontaneously post-HCT, ${ }^{16}$ it is unlikely that the longer duration of prophylaxis alone was responsible for our results. Finally, due to the slow uptake of the new strategy, we were unable to perform a before-vs-after policy change analysis. However, there does appear to be a year-over-year reduction in cumulative incidence of VZV disease and PHN for all patients (supplemental Table 3).

This study also raises questions for future study. Notably, what is the degree and persistence of cellular immune response to varicella vaccination post-HCT in adults, and what clinical/immunological factors affect this? In addition, the optimal timing of vaccination post-HCT should be studied: for example, we plan to examine prospectively whether the vaccine can be delivered safely and effectively after 1 year of acyclovir/valacyclovir prophylaxis.

In conclusion, we suggest that the combination of a VZV prophylaxis strategy that includes 2 years of acyclovir/valacyclovir prophylaxis followed by 2 doses of the live attenuated varicella vaccine is a safe

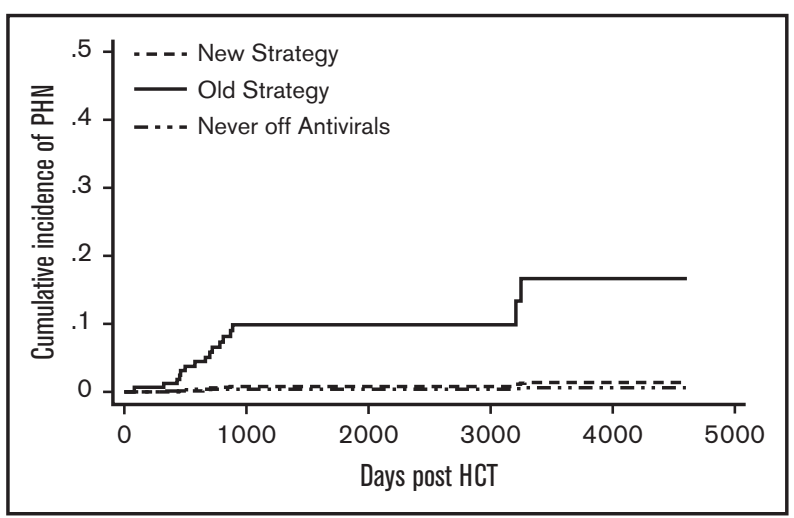

Figure 3. Cumulative incidence of PHN. The difference between the new strategy and the old strategy groups was significant $(P=.02)$. The never off antivirals group consisted of patients who were on acyclovir/valacyclovir until the end of follow-up. 
and effective way of reducing the incidence of VZV disease and $\mathrm{PHN}$ in adult HCT recipients.

\section{Acknowledgments}

The authors appreciate the help of Alberta provincial and regional public health zone staff, including Donna Jones, Tania Weisberger, Cindy Dribnenki, Margo Kirkwood, Joan Ing, Chelsea Morrow, Elizabeth Colangelo, Winson Wong, Tara Harpe, Donna Koch, Sjaane Heikoop, Terri Forbis, Tom Baker, and the Cross Cancer Centre health records staff, including Vanessa Benjamin, Lori Kotkas, and Kelsi McKinnon, and the members of the Alberta Blood and Marrow Transplant Program, including Nizar Bahlis, Christopher Brown, Michelle Geddes, Lynne Savoie, Mona Shafey, Minakshi Taparia, Nan Cox-Kennett, and Anne Tremblay.

\section{Authorship}

Contribution: K.J. designed the study, collected and analyzed the data, and prepared the manuscript; J.M. and M.L. assisted in procuring public health vaccine data and critically reviewed the manuscript; T.S.W., C.B.B., A.C., V.H.J.-Z., P.D., J.T., D.S., and A.D. critically reviewed the manuscript; J.S. developed the new prophylaxis strategy, conceived the research question, designed the study, supervised data analyses, and critically reviewed/edited the manuscript.

Conflict-of-interest disclosure: The authors declare no competing financial interests.

Correspondence: Kareem Jamani, Room CC110c, Tom Baker Cancer Centre, 133129 St NW, Calgary, AB T2N 4N2, Canada; e-mail: kareem.jamani@ahs.ca.

\section{References}

1. Ho DY, Arvin AM. Varicella Zoster Virus Infections. In: Forman SJ, Negrin RS, Antin JH, Appelbaum FR, eds. Thomas' Hematopoietic Cell Transplantation, 5th ed. Hoboken, NJ: Wiley-Blackwell; 2016

2. Marr KA. Delayed opportunistic infections in hematopoietic stem cell transplantation patients: a surmountable challenge. Hematology/the Education Program of the American Society of Hematology. American Society of Hematology. Education Program. 2012;2012:265-270.

3. Rogers JE, Cumpston A, Newton M, Craig M. Onset and complications of varicella zoster reactivation in the autologous hematopoietic cell transplant population. Transplant infectious disease: an Official Journal of the Transplantation Society. 2011;13(5):480-484.

4. Blennow O, Fjaertoft G, Winiarski J, Ljungman P, Mattsson J, Remberger M. Varicella-zoster reactivation after allogeneic stem cell transplantation without routine prophylaxis-the incidence remains high. Biology of Blood and Marrow Transplantation: Journal of the American Society for Blood and Marrow Transplantation. 2014;20(10):1646-1649.

5. Doki N, Miyawaki S, Tanaka M, et al. Visceral varicella zoster virus infection after allogeneic stem cell transplantation. Transplant Infectious Disease: an Official Journal of the Transplantation Society. 2013;15(3):314-318.

6. Koc Y, Miller KB, Schenkein DP, et al. Varicella zoster virus infections following allogeneic bone marrow transplantation: frequency, risk factors, and clinical outcome. Biology of Blood and Marrow Transplantation: Journal of the American Society for Blood and Marrow Transplantation. 2000;6(1):44-49.

7. Locksley RM, Flournoy N, Sullivan KM, Meyers JD. Infection with varicella-zoster virus after marrow transplantation. J Infect Dis. $1985 ; 152(6): 1172-1181$.

8. Offidani $M$, Corvatta $L$, Olivieri $A$, et al. A predictive model of varicella-zoster virus infection after autologous peripheral blood progenitor cell transplantation. Clin Infect Dis. 2001;32(10):1414-1422.

9. Ljungman $\mathrm{P}$, Wilczek $\mathrm{H}$, Gahrton $\mathrm{G}$, et al. Long-term acyclovir prophylaxis in bone marrow transplant recipients and lymphocyte proliferation responses to herpes virus antigens in vitro. Bone Marrow Transplant. 1986;1(2):185-192.

10. Perren TJ, Powles RL, Easton D, Stolle K, Selby PJ. Prevention of herpes zoster in patients by long-term oral acyclovir after allogeneic bone marrow transplantation. Am J Med. 1988;85(2A):99-101.

11. Selby PJ, Powles RL, Easton $D$, et al. The prophylactic role of intravenous and long-term oral acyclovir after allogeneic bone marrow transplantation. Br $J$ Cancer. 1989;59(3):434-438.

12. Boeckh M, Kim HW, Flowers ME, Meyers JD, Bowden RA. Long-term acyclovir for prevention of varicella zoster virus disease after allogeneic hematopoietic cell transplantation-a randomized double-blind placebo-controlled study. Blood. 2006;107(5):1800-1805.

13. Thomson KJ, Hart DP, Banerjee L, Ward KN, Peggs KS, Mackinnon S. The effect of low-dose aciclovir on reactivation of varicella zoster virus after allogeneic haemopoietic stem cell transplantation. Bone Marrow Transplant. 2005;35(11):1065-1069.

14. Erard V, Guthrie KA, Varley C, et al. One-year acyclovir prophylaxis for preventing varicella-zoster virus disease after hematopoietic cell transplantation: no evidence of rebound varicella-zoster virus disease after drug discontinuation. Blood. 2007;110(8):3071-3077.

15. Pollack $\mathrm{M}$, Heugel $\mathrm{J}, \mathrm{Xie} \mathrm{H}$, et al. An international comparison of current strategies to prevent herpesvirus and fungal infections in hematopoietic cel transplant recipients. Biology of Blood and Marrow Transplantation: Journal of the American Society for Blood and Marrow Transplantation. 2011; 17(5):664-673.

16. Distler E, Schnurer E, Wagner E, et al. Recovery of varicella-zoster virus-specific $T$ cell immunity after $T$ cell-depleted allogeneic transplantation requires symptomatic virus reactivation. Biology of Blood and Marrow Transplantation: Journal of the American Society for Blood and Marrow Transplantation. 2008;14(12):1417-1424.

17. Chou JF, Kernan NA, Prockop S, et al. Safety and immunogenicity of the live attenuated varicella vaccine following T replete or T cell-depleted related and unrelated allogeneic hematopoietic cell transplantation (alloHCT). Biology of Blood and Marrow Transplantation: Journal of the American Society for Blood and Marrow Transplantation. 2011;17(11):1708-1713.

18. Kussmaul SC, Horn BN, Dvorak CC, Abramovitz L, Cowan MJ, Weintrub PS. Safety of the live, attenuated varicella vaccine in pediatric recipients of hematopoietic SCTs. Bone Marrow Transplant. 2010;45(11):1602-1606. 
19. Sauerbrei A, Prager J, Hengst U, Zintl F, Wutzler P. Varicella vaccination in children after bone marrow transplantation. Bone Marrow Transplant. 1997; 20(5):381-383.

20. Ljungman P, Cordonnier C, Einsele H, et al; Center for International Blood and Marrow Transplant Research; National Marrow Donor Program; European Blood and Marrow Transplant Group; American Society of Blood and Marrow Transplantation; Canadian Blood and Marrow Transplant Group; Infectious Disease Society of America; Society for Healthcare Epidemiology of America; Association of Medical Microbiology and Infectious Diseases Canada; Centers for Disease Control and Prevention. Vaccination of hematopoietic cell transplant recipients. Bone Marrow Transplant. 2009;44(8):521-526.

21. Public Health \& Compliance Division AH. Immunization for Adult Hematopoietic Stem Cell Transplant Recipients. Alberta Immunization Policy-Special Situations for Immunization 2007; http://www.health.alberta.ca/documents/AIP-Special-Situations-Adult-Hematopoietic-Stem-Cell-Transplant-HSCT. pdf. Accessed September 16, 2016.

22. Russell JA, Duan Q, Chaudhry MA, et al. Transplantation from matched siblings using once-daily intravenous busulfan/fludarabine with thymoglobulin: a myeloablative regimen with low nonrelapse mortality in all but older patients with high-risk disease. Biology of Blood and Marrow Transplantation: Journal of the American Society for Blood and Marrow Transplantation. 2008;14(8):888-895.

23. Russell JA, Irish W, Balogh A, et al. The addition of $400 \mathrm{cGY}$ total body irradiation to a regimen incorporating once-daily intravenous busulfan, fludarabine, and antithymocyte globulin reduces relapse without affecting nonrelapse mortality in acute myelogenous leukemia. Biology of Blood and Marrow Transplantation: Journal of the American Society for Blood and Marrow Transplantation. 2010;16(4):509-514.

24. Przepiorka D, Weisdorf D, Martin P, et al. 1994 Consensus Conference on Acute GVHD Grading. Bone Marrow Transplant. 1995;15(6):825-828.

25. Shulman HM, Sullivan KM, Weiden PL, et al. Chronic graft-versus-host syndrome in man. A long-term clinicopathologic study of 20 Seattle patients. Am J Med. 1980;69(2):204-217.

26. Filipovich $\mathrm{AH}$, Weisdorf $\mathrm{D}$, Pavletic $\mathrm{S}$, et al. National Institutes of Health consensus development project on criteria for clinical trials in chronic graftversus-host disease: I. Diagnosis and staging working group report. Biology of Blood and Marrow Transplantation: Journal of the American Society for Blood and Marrow Transplantation. 2005;11(12):945-956.

27. Nalamachu S, Morley-Forster P. Diagnosing and managing postherpetic neuralgia. Drugs Aging. 2012;29(11):863-869.

28. Boeckh M. Prevention of VZV infection in immunosuppressed patients using antiviral agents. Herpes: the Journal of the IHMF. 2006;13(3):60-65.

29. Arai S, Arora M, Wang T, et al. Increasing incidence of chronic graft-versus-host disease in allogeneic transplantation: a report from the Center for International Blood and Marrow Transplant Research. Biology of Blood and Marrow Transplantation: Journal of the American Society for Blood and Marrow Transplantation. 2015;21(2):266-274.

30. Remberger M, Beelen DW, Fauser A, Basara N, Basu O, Ringdén O. Increased risk of extensive chronic graft-versus-host disease after allogeneic peripheral blood stem cell transplantation using unrelated donors. Blood. 2005;105(2):548-551.

31. Steer CB, Szer J, Sasadeusz J, Matthews JP, Beresford JA, Grigg A. Varicella-zoster infection after allogeneic bone marrow transplantation: incidence, risk factors and prevention with low-dose aciclovir and ganciclovir. Bone Marrow Transplant. 2000;25(6):657-664 .

32. Johnson RW, Bouhassira D, Kassianos G, Leplège A, Schmader KE, Weinke T. The impact of herpes zoster and post-herpetic neuralgia on quality-of-life. BMC Med. 2010;8:37-49.

33. Serpell M, Gater A, Carroll S, Abetz-Webb L, Mannan A, Johnson R. Burden of post-herpetic neuralgia in a sample of UK residents aged 50 years or older: findings from the Zoster Quality of Life (ZQOL) study. Health Qual Life Outcomes. 2014;12:92-105.

34. Oxman MN, Levin MJ, Johnson GR, et al; Shingles Prevention Study Group. A vaccine to prevent herpes zoster and postherpetic neuralgia in older adults. N Engl J Med. 2005;352(22):2271-2284.

35. Galea SA, Sweet A, Beninger P, et al. The safety profile of varicella vaccine: a 10-year review. J Infect Dis. 2008;197(suppl 2):S165-S169.

36. Michalik DE, Steinberg SP, Larussa PS, et al. Primary vaccine failure after 1 dose of varicella vaccine in healthy children. J Infect Dis. 2008;197(7): 944-949.

37. Helmuth IG, Poulsen A, Suppli CH, Mølbak K. Varicella in Europe-a review of the epidemiology and experience with vaccination. Vaccine. 2015;33(21): 2406-2413.

38. Nader S, Bergen R, Sharp M, Arvin AM. Age-related differences in cell-mediated immunity to varicella-zoster virus among children and adults immunized with live attenuated varicella vaccine. J Infect Dis. 1995;171(1):13-17.

39. Tomblyn M, Chiller T, Einsele H, et al. Guidelines for preventing infectious complications among hematopoietic cell transplantation recipients: a global perspective. Biology of Blood and Marrow Transplantation: Journal of the American Society for Blood and Marrow Transplantation. 2009;15(10):11431238.

40. Bosch M, Dhadda M, Hoegh-Petersen M, et al. Immune reconstitution after anti-thymocyte globulin-conditioned hematopoietic cell transplantation. Cytotherapy. 2012;14(10):1258-1275.

41. Gershon AA, Steinberg SP. Cellular and humoral immune responses to varicella-zoster virus in immunocompromised patients during and after varicellazoster infections. Infect Immun. 1979;25(1):170-174.

42. Burke BL, Steele RW, Beard OW, Wood JS, Cain TD, Marmer DJ. Immune responses to varicella-zoster in the aged. Arch Intern Med. 1982;142(2): 291-293.

43. Ludwig B, Kraus FB, Allwinn R, Keim S, Doerr HW, Buxbaum S. Loss of varicella zoster virus antibodies despite detectable cell mediated immunity after vaccination. Infection. 2006;34(4):222-226. 